Pacific Journal of Mathematics

STRONGLY SEMISIMPLE ABELIAN GROUPS 


\title{
STRONGLY SEMISIMPLE ABELIAN GROUPS
}

\author{
R. A. Beaumont and D. A. Lawver
}

For an abelian group $G$ and a ring $R, R$ is a ring on $G$ if the additive group of $R$ is isomorphic to $G$. $G$ is nil if the only ring $R$ on $G$ is the zero ring, $R^{2}=\{0\} . \quad G$ is radical if there is a nonzero ring on $G$ that is radical in the Jacobson sense. Otherwise, $G$ is antiradical. $G$ is semisimple if there is some (Jacobson) semisimple ring on $G$, and $G$ is strongly semisimple if $G$ is nonnil and every nonzero ring on $G$ is semisimple. It is shown that the only strongly semisimple torsion groups are cyclic of prime order, and that no mixed group is strongly semisimple. The torsion free rank one strongly semisimple groups are characterized in terms of their type, and it is shown that the strongly semisimple and antiradical rank one groups coincide. For torsion free groups it is shown that the property of being strongly semisimple is invariant under quasi-isomorphism and that a strongly semisimple group is strongly indecomposable. Further, for a strongly indecomposable torsion free group $G$ of finite rank, the following are equivalent: (a) $G$ is semisimple, (b) $G$ is strongly semisimple, (c) $G \cong R^{+}$where $R$ is a full subring of an algebraic number field $K$ such that $[K, Q]=\operatorname{rank} G$ where $Q$ is the field of rational numbers and $R \doteq J_{\pi}$, where $\pi$ is either empty or an infinite set of primes in $K,(d) G$ is nonnil and antiradical.

Introduction. In [4], F. Haimo considered the problem of characterizing those abelian groups $G$ that are the additive groups of nontrivial radical rings, where the radical under consideration is the Jacobson radical. It was observed by the present authors that for several classes of groups, those groups $G$ that did not support nontrivial radical rings (antiradical groups) satisfied a much stronger condition, namely, that every nontrivial ring on $G$ is semisimple (strongly semisimple groups). This suggested the problem of identifying classes of groups for which the antiradical and strongly semisimple groups coincide, and the problem of characterizing strongly semisimple groups.

Section 1 contains the basic definitions. The case of torsion and mixed groups is disposed of in $\S 2$ where it is shown that the only strongly semisimple torsion groups are the cyclic groups of prime order, and that no mixed group is strongly semisimple. In $\S 3$, the torsion free rank one strongly semisimple groups are characterized in terms of their type, and it is shown that the strongly semisimple and antiradical groups coincide. In $\S 4$, it is shown that the property 
of being strongly semisimple for a torsion free group is invariant under quasi-isomorphism and that a strongly semisimple torsion free group is strongly indecomposable. Applying results on torsion free rings in [1], [2], and [6], we show in $\S 5$, that the results for rank one can be recovered for strongly indecomposable torsion free groups of finite rank.

Throughout the paper, group means additive abelian group and ring means associative ring. The notation is standard and generally follows that of [3]. The field of rational numbers as well as its additive group is denoted by $Q, Z$ denotes the integers, and $\mathscr{J}(R)$ is the Jacobson radical of a ring $R$.

1. Definitions. For any ring $R$, let $\mathscr{N}(R)$ denote the sum of all nilpotent left ideals. $\mathscr{N}(R)$ is a nil ideal and $\mathscr{N}(R)$ contains all nilpotent right ideals. Let $\mathscr{J}(R)$ denote the Jacobson radical of $R$ [5]. Then $\mathcal{J}(R) \supseteq \mathscr{N}(R)$. If $G$ is any group, then $R$ is a ring on $G$ if $R^{+}$, the additive group of $R$, is isomorphic to $G$. The zero ring on $G$ is the ring obtained by defining $x \cdot y=0$ for all $x, y \in G$. If $R$ is the zero ring on $G$, then $R^{2}=\{0\}$, and $R=\mathscr{J}(R)=\mathscr{N}(R)$. A group $G$ is a nil group if the only ring on $G$ is the zero ring. Otherwise $G$ is nonnil.

Definition 1.1. (Haimo, [4]) A group $G$ is a radical group if there is a ring $R$ on $G$ such that $R=\mathscr{J}(R)$ and $R$ is not the zero ring on $G$. Otherwise, $G$ is an antiradical group.

Definition 1.2. A group $G \neq\{0\}$ is a semisimple group if there is a ring $R$ on $G$ such that $\mathscr{J}(R)=\{0\}$.

DEFINITION 1.3. A group $G$ is a strongly semisimple group if $G$ is nonnil and $\mathscr{J}(R)=\{0\}$ for every nonzero ring $R$ on $G$.

We note that a nil group is antiradical, a semisimple group is nonnil and that a strongly semisimple group is semisimple and antiradical. Moreover, if there is a nonzero ring $R$ on $G$ such that $\mathscr{N}(R) \neq\{0\}$, then $G$ is not strongly semisimple.

The cyclic group of order six, $Z(6)$, is semisimple, antiradical, and not strongly semisimple. The direct sum of $2^{\aleph_{0}}$ copies of the additive group of rational numbers, $Q$, is radical and semisimple [4].

The following simple observation will be useful.

Lemma 1.4. If $G=H \oplus K, H \neq\{0\}, K \neq\{0\}$, and either $H$ or $K$ is nonnil, then there is a nonzero ring $R$ on $G$ such that $\mathscr{N}(R) \neq\{0\}$. 
Proof. Suppose $H$ is nonnil and let $R_{H}$ be a nonzero ring on $H$. Let $R_{K}$ be the zero ring on $K$. Then the ring direct sum $R=R_{H}+$ $R_{K}$ is a nonzero ring on $H \oplus K$ such that $\mathscr{N}(R) \supseteqq R_{K} \neq\{0\}$.

2. Torsion and mixed groups. As mentioned in the introduction, our purpose is to characterize strongly semisimple groups. This is easily done if $G$ is torsion group or a mixed group.

By Theorem 69.3 [3], there is a ring $R$ on a torsion group $G$ with $\mathscr{N}(R)=\{0\}$ if and only if $G$ is an elementary group. Since an elementary group $G \neq\{0\}$ is the additive group of a direct sum of fields, there is a ring on $G$ with $\mathscr{J}(R)=\{0\}$. Thus, a torsion group $G$ is a semisimple group if and only if $G$ is elementary.

THEOREM 2.1. The only strongly semisimple torsion groups are the cyclic groups of order $p, Z(p), p$ a prime.

Proof. If $G$ is strongly semisimple, then $G$ is semisimple, and by the above remarks, $G$ is the direct sum of cyclic groups of order $p$ for various primes $p$. Since the groups $Z(p)$ are nonnil, it follows from 1.4 that if the direct decomposition of $G$ has more than one component, then $G$ is not strongly semisimple. On the other hand, every nonzero ring on $Z(p)$ is isomorphic to the field with $p$ elements. Thus, $Z(p)$ is strongly semisimple.

THEOREM 2.2. If $G$ is a mixed group, then there is a nonzero ring $R$ on $G$ such that $\mathscr{N}(R) \neq\{0\}$.

Proof. Suppose first that $G$ is not reduced. If the maximal divisible subgroup, $G_{d}$, of $G$ is not torsion, then $G=Q \oplus G_{1}$, where $G_{1} \neq\{0\}$. By 1.4, there is a nonzero ring $R$ on $G$ with $\mathscr{N}(R) \neq$ $\{0\}$. If $G_{d}$ is torsion, then since $G_{d}$ is a nil group and $G_{d}$ is an ideal in any ring $R$ on $G, \mathscr{N}(R) \supseteqq G_{d} \neq\{0\}$ for any ring $R$ on $G$. Since $G$ is mixed there is a nonzero ring $R$ on $G$. On the other hand, if $G$ is reduced, then $G=\{x\} \oplus G_{2}$, where $\{x\}$ is a finite cyclic group and $G_{2} \neq\{0\}$. Since $\{x\}$ is nonnil, it again follows from 1.4 that there is a ring $R$ on $G$ with $\mathscr{N}(R) \neq\{0\}$.

COROLlaRY 2.3. No mixed group is strongly semisimple.

3. Rank one torsion free groups. We first characterize the strongly semisimple torsion free groups of rank 1 in terms of their types. If $G$ is a rank 1 group, we write the type of $G$ as $T(G)=$ $\left[\left(k_{1}, k_{2}, \cdots, k_{n}, \cdots\right)\right]$, where $\left(k_{1}, k_{2}, \cdots, k_{n}, \cdots\right)$ is the height of a nonzero element $g \in G$; that is, for the prime $p_{n}, k_{n}$ is the $p_{n}$-height of $g$. 
Let $\pi$ be an arbitrary set of primes, and let $m$ be a fixed positive integer such that $(m, p)=1$ for every $p \in \pi$. Denote by $S(m, \pi)$ that subring of $Q$ consisting of all rational numbers of the form $\mathrm{mr} / \mathrm{s}$, where $s$ is a product of primes in $\pi$ and $r$ is any integer. Let $\pi^{c}$ be the complement of $\pi$ in the set of all primes, and let $\pi^{\prime}$ be the set of all primes $p \in \pi^{c}$ such that $(m, p)=1$.

$$
\begin{aligned}
& \text { Lemma 3.1. } \mathscr{J}(S(m, \pi))=\bigcap_{p \in \pi^{\prime}} p S(m, \pi) \text { if } \pi^{c} \neq \phi . \\
& \mathcal{J}(S(m, \pi))=\{0\} .
\end{aligned}
$$

Proof. If $\pi^{c}=\phi$, then $m=1$ and $S(m, \pi)=Q$. Thus, $\mathcal{J}(S(m$, $\pi))=\{0\}$. We show that if $\pi^{c} \neq \phi$, then $\left\{p S(m, \pi) \mid p \in \pi^{\prime}\right\}$ is the collection of maximal modular ideals in $S(m, \pi)$. Since $p \in \pi^{\prime} \subseteq \pi^{c}$, the ideal $p S(m, \pi) \neq S(m, \pi)$, e.g., $m \notin p S(m, \pi)$. If $m r / s \in S(m, \pi)$ and $m r / s \notin$ $p S(m, \pi)$, we have $(r, p)=1$. Then $x r+y p=1$ for $x, y \in Z$, and $x m r+y m p=m$. Thus, $m$ is in the ideal generated by $p S(m, \pi)$ and $m r / s$. That is, this ideal is $S(m, \pi)$. Hence $p S(m, \pi)$ is maximal. Since $p \in \pi^{\prime},(m, p)=1$. Thus, $x m+y p=1$ for $x, y \in Z$. If $m r / s \in$ $S(m, \pi)$, then $(x m)(m r / s)+(y p)(m r / s)=m r / s$, or $m r / s-(x m)(m r / s)=$ pymr $/ s \in p S(m, \pi)$. Hence $x m$ is an identity modulo $p S(m, \pi)$. Therefore, $p S(m, \pi)$ is modular.

Suppose that $\mathscr{F} \neq\{0\}$ is an ideal in $S(m, \pi)$. If $m r$ is the least positive integer in $\mathscr{F}$, then every element of $\mathscr{F}$ is a multiple of $m r$. Note that $(m r, p)=1$ for $p \in \pi$. If $r=1, \mathscr{J}=S(m, \pi)$. If $r \neq 1$, $\mathscr{J} \subseteq r S(m, \pi) \subseteq p S(m, \pi)$ for some $p \in \pi^{c}$. Thus, the maximal ideals in $S(m, \pi)$ are the ideals $p S(m, \pi)$ for $p \in \pi^{c}$. If $p S(m, \pi)$ is modular, then in particular, there is an element $m r / s \in S(m, \pi)$ such that $m$ $(m r / s) m=p m r^{\prime} / s^{\prime} \in p S(m, \pi)$. This equation yields $s s^{\prime}-m r s^{\prime}=p r^{\prime} s$. Since $\left(s s^{\prime}, p\right)=1$ for $p \in \pi^{c}$, it follows that $(m, p)=1$. That is, $p \in \pi^{\prime}$.

Note that if $\pi^{\prime}=\phi$, then the collection of maximal modular ideals is vacuous and $\mathscr{J}(S(m, \pi))=S(m, \pi)=\bigcap_{p \in \pi^{\prime}} p S(m, \pi)[5$, p. 9].

THEOREM 3.2. Let $G$ be a torsion free group of rank 1. Then the following statements are equivalent:

(a) $G$ is semisimple.

(b) $G$ is strongly semisimple.

(c) $T(G)=\left[\left(k_{1}, k_{2}, \cdots, k_{n}, \cdots\right)\right]$, where $k_{n}=0$ or $\infty$ for all $n$, and either $k_{n}=\infty$ for all $n$ or $k_{n}=0$ for infinitely many $n$.

(d) $G$ is nonnil and antiradical.

Proof. It follows from Definitions 1.2 and 1.3 that (b) implies (a).

To prove that (c) implies (b), we note that since $k_{n}=0$ or $\infty$ for all $n$, it follows [3, p. 269] that $G$ is nonnil and any nonzero 
ring $R$ on $G$ is isomorphic to a subring $S(m, \pi)$ of $Q$, where $\pi$ is the set of all primes for which $k_{n}=\infty$. If $k_{n}=\infty$ for all $n$, then $R \cong$ $S(m, \pi)=Q$, so that $G$ is strongly semisimple. If $k_{n}=0$ for infinitely many $n$, then $\pi^{c}$ is infinite, so that $\pi^{\prime}$, the set of all primes $p$ in $\pi^{c}$ such that $(m, p)=1$, is also infinite. If $m r / s \in \bigcap_{p \in \pi^{\prime}} p S(m, \pi)$, then $p \mid r$ for all $p \in \pi^{\prime}$. Hence, $m r / s=0$. By Lemma 3.1, $\mathscr{J}(S(m, \pi))=$ $\{0\}$. Therefore, $G$ is strongly semisimple.

We next show that (a) implies (c). Assume that (c) is not satisfied. Then either $0<k_{n}<\infty$ for infinitely many $n$, or $k_{n}=0$ or $\infty$ for all $n$ and $k_{n}=\infty$ for almost all $n$, but not all $n$. In the first case $G$ is a nil group, and therefore not semisimple. In the second case, any ring $R$ on $G$ is isomorphic to an $S(m, \pi)$, where $\pi^{c}$ is finite and not empty. Therefore, $\pi^{\prime}$ is finite. By Lemma 3.1, $\mathscr{J}(S(m, \pi))=$ $\bigcap_{p_{\in},} p S(m, \pi)=S(m, \pi)$ if $\pi^{\prime}=\phi$, and $\mathscr{J}(S(m, \pi))=p_{1} p_{2} \cdots p_{k} S(m$, $\pi) \neq\{0\}$ if $\pi^{\prime}=\left\{p_{1}, p_{2}, \cdots, p_{k}\right\} \neq \phi$. Therefore, $G$ is not semisimple.

Since $(b) \Rightarrow(d)$ by Definitions 1.1 and 1.3 , we complete the chain of implications, by showing that (d) implies (c). Here we observe from the above argument that if (c) is not satisfied, then either $G$ is a nil group, or any ring $R$ on $G$ is either radical or $p_{1} p_{2} \cdots p_{k} R$ is radical. But since $G \cong R^{+} \cong\left(p_{1} p_{2} \cdots p_{k} R\right)^{+}, G$ is isomorphic to a radical group, and hence is radical. Haimo [4, Theorem 4] proves that (c) and (d) are equivalent in a somewhat different manner.

\section{COROLLARY 3.3. $Z$ and $Q$ are strongly semisimple.}

4. Quasi-isomorphism. We show that for torsion free groups the property of being strongly semisimple is invariant under quasiisomorphism. This follows from Thorem 2.6 and Corollary 2.7 in [2]. Theorem 2.6 in [2] states that if $G$ and $H$ are quasi-isomorphic, and if $R$ is a ring on $G$, then there is a ring $S$ on $H$ and a positive integer $n$ such that $S$ is isomorphic to a subring $T$ of $R$ and $n R \cong$ $T$. Corollary 2.7 in [2] states that if $G$ and $H$ are quasi-isomorphic and $R$ is a ring on $G$, then there is a ring $S$ on $H$ such that the rational algebras $Q \otimes_{z} R$ and $Q \otimes_{z} S$ are isomorphic. It follows at once from this result that if $G$ and $H$ are quasi-isomorphic and $G$ is a nil group, then $H$ is a nil group.

THEOREM 4.1. Let $G$ and $H$ be quasi-isomorphic torsion free groups. If $G$ is strongly semisimple, then so is $H$.

Proof. Assume that $G$ is strongly semisimple and $H$ is not strongly semisimple. Then either $H$ is nil or there is a nonzero ring $R$ on $H$ such that $\mathscr{J}(R) \neq\{0\}$. If $H$ is nil, then $G$ is nil and hence not strongly semisimple. In the second case, it follows from the above 
remarks, that there is a nonzero ring $S$ on $G$ such that $S$ is isomorphic to a subring $T$ of $R$ and $n R \subseteq T$ for some positive integer $n$. Now $n \mathscr{J}(R)$ is an ideal in $R$ and $n \mathscr{J}(R) \subseteq n R \subseteq T$. Thus, $n \mathcal{J}(R)$ is an ideal in $T$. Therefore, $\mathscr{J}(T) \supseteqq \mathscr{J}(T) \cap n \mathscr{J}(R)=\mathscr{J}(n \mathscr{J}(R))$. Moreover, $\mathscr{J}(n \mathscr{J}(R))=\mathscr{J}(R) \cap n \mathcal{J}(R)=n \mathscr{J}(R)$. Hence $\mathscr{J}(T) \supseteqq$ $n \mathcal{J}(R) \neq\{0\}$. Therefore, $T$ is not semisimple, and consequently $S$ is not semisimple. This contradicts the hypothesis that $G$ is strongly semisimple.

A torsion free group $G$ is strongly indecomposable if whenever $G$ is quasi-isomorphic to a direct sum $G_{1} \oplus G_{2}, G_{1}$ and $G_{2}$ torsion free, then either $G_{1}=\{0\}$ or $G_{2}=\{0\}$. Otherwise $G$ is quasi-decomposable.

THEOREM 4.2. A strongly semisimple torsion free group $G$ is strongly indecomposable.

Proof. Assume that $G$ is quasi-decomposable. Then $G$ is quasiisomorphic to $G_{1} \oplus G_{2}$, where $G_{1}$ and $G_{2}$ are nonzero torsion free groups. By Theorem 4.1, $G_{1} \oplus G_{2}$ is strongly semisimple. If either $G_{1}$ or $G_{2}$ is nonnil, then by Lemma 1.4, $G_{1} \oplus G_{2}$ is not strongly semisimple. Hence we may assume that both $G_{1}$ and $G_{2}$ are nil groups. Moreover, $G_{1} \oplus G_{2}$, being strongly semisimple, is nonnil.

Let $*$ be a nontrivial associative multiplication on $G_{1} \oplus G_{2}$. Let $\pi_{G_{1}}$ and $\pi_{G_{2}}$ be the projections of $G_{1} \oplus G_{2}$ onto $G_{1}$ and $G_{2}$, respectively. For $\left(x_{1}, y_{1}\right),\left(x_{2}, y_{2}\right)$ in $G_{1} \oplus G_{2}$ define a multiplication $\circ$ on $G_{1} \oplus G_{2}$ by $\left(x_{1}, y_{1}\right) \circ\left(x_{2}, y_{2}\right)=\left(0, \pi_{G_{2}}\left[\left(x_{1}, 0\right) *\left(x_{2}, 0\right)\right]\right)$. If $\pi_{G_{2}}\left[\left(x_{1}, 0\right) *\left(x_{2}, 0\right)\right] \neq 0$ for some $x_{1}, x_{2} \in G_{1}$, then $\circ$ is an associative multiplication on $G_{1} \oplus G_{2}$ such that $\left(G_{1} \oplus G_{2}\right)^{2} \neq\{0\}$ and $\left(G_{1} \oplus G_{2}\right)^{3}=\{0\}$. Therefore, $G_{1} \oplus G_{2}$ is a radical group, contradicting the fact that $G_{1} \oplus G_{2}$ is strongly semisimple. If $\pi_{G_{2}}\left[\left(x_{1}, 0\right)^{*}\left(x_{2}, 0\right)\right]=0$ for all $x_{1}, x_{2} \in G_{1}$, define $\left(x_{1}, y_{1}\right) \times\left(x_{2}, y_{2}\right)=\left(\pi_{G_{1}}[(0\right.$, $\left.\left.\left.y_{1}\right)^{*}\left(0, y_{2}\right)\right], 0\right)$. As above, if $\pi_{G_{1}}\left[\left(0, y_{1}\right)^{*}\left(0, y_{2}\right)\right] \neq 0$ for some $y_{1}, y_{2} \in G_{2}$, $\times$ is an associative multiplication on $G_{1} \oplus G_{2}$ such that $\left(G_{1} \oplus G_{2}\right)^{2} \neq$ $\{0\}$ and $\left(G_{1} \oplus G_{2}\right)^{3}=\{0\}$, again contradicting the fact that $G_{1} \oplus G_{2}$ is strongly semisimple.

We may now assume that $\pi_{G_{2}}\left[\left(x_{1}, 0\right)^{*}\left(x_{2}, 0\right)\right]=0$ for all $x_{1}, x_{2} \in G_{1}$ and that $\pi_{G_{1}}\left[\left(0, y_{1}\right)^{*}\left(0, y_{2}\right)\right]=0$ for all $y_{1}, y_{2} \in G_{2}$. It follows that $\left(x_{1}\right.$, $\left.0)^{*}\left(x_{2}, 0\right)=\left(x\left(x_{1}, x_{2}\right), x_{1}\right), 0\right)$ for all $x_{1}, x_{2} \in G_{1}$ and that $\left(0, y_{1}\right)^{*}\left(0, y_{2}\right)=$ $\left(0, y\left(y_{1}, y_{2}\right)\right)$ for all $y_{1}, y_{2} \in G_{2}$. Then $x_{1}{ }^{*} x_{2}=x\left(x_{1}, x_{2}\right)$ and $y_{1}{ }^{*} y_{2}=y\left(y_{1}, y_{2}\right)$ are associative multiplications on $G_{1}$ and $G_{2}$, respectively. Since $G_{1}$ and $G_{2}$ are nil groups, $x\left(x_{1}, x_{2}\right)=y\left(y_{1}, y_{2}\right)=0$ for all $x_{1}, x_{2} \in G_{1}$ and all $y_{1}, y_{2} \in G_{2}$. That is, $\left(x_{1}, 0\right) *\left(x_{2}, 0\right)=(0,0)$ and $\left(0, y_{1}\right)^{*}\left(0, y_{2}\right)=(0,0)$ for all $x_{1}, x_{2} \in G_{1}$ and all $y_{1}, y_{2} \in G_{2}$, so that under the multiplication *, $G_{1}$ and $G_{2}$ are subrings of $G_{1} \oplus G_{2}$ such that $G_{1}^{2}=\{0\}$ and $G_{2}^{2}=\{0\}$.

Let $r\left(G_{1}\right)$ and $r\left(G_{2}\right)$ be the right annililators of $G_{1}$ and $G_{2}$, respectively in the ring $G_{1} \oplus G_{2}$ with multiplication *. Then $r\left(G_{1}\right) \supseteqq G_{1}$ and 
$r\left(G_{2}\right) \supseteqq G_{2}$. If $r\left(G_{1}\right)=G_{1}$, then $r\left(G_{1}\right)$ is a nonzero nilpotent right ideal in $G_{1} \oplus G_{2}$. Hence $\mathcal{J}\left(G_{1} \oplus G_{2}\right) \supseteqq r\left(G_{1}\right)=G_{1} \neq\{0\}$. Therefore, the group $G_{1} \oplus G_{2}$ is not strongly semisimple, contrary to hypothesis. If $r\left(G_{1}\right) \supset$ $G_{1}$, let $g \in r\left(G_{1}\right), g \notin G_{1}$. Since $r\left(G_{1}\right) \supseteqq G_{1}$ and $r\left(G_{2}\right) \supseteqq G_{2}, r\left(G_{1}\right)+r\left(G_{2}\right)=$ $G_{1} \oplus G_{2}$. Therefore, $g=g_{1}+g_{2}$, where $g_{1} \in G_{1}, g_{2} \in G_{2}$, and $g_{2} \neq 0$. Thus, $g_{2}=g-g_{1} \in r\left(G_{1}\right) \cap G_{2}$. It follows that the infinite cyclic group $\left(g_{2}\right)$ is a nonzero nilpotent left ideal in $G_{1} \oplus G_{2}$. Indeed, if $x+y \in G_{1} \oplus$ $G_{2}, x \in G_{1}, y \in G_{2}$, then $(x+y)^{*} g_{2}=x^{*} g_{2}+y^{*} g_{2}=0+0=0$, since $g_{2} \in$ $r\left(G_{1}\right) \cap G_{2}$. Therefore, $\mathscr{J}\left(G_{1} \oplus G_{2}\right) \supseteqq\left(g_{2}\right) \neq\{0\}$, and again we have contradicted the fact that $G_{1} \oplus G_{2}$ is strongly semisimple, completing the proof.

5. Strongly indecomposable torsion free groups. Theorem 4.2 allows us to restrict our attention to strongly indecomposable torsion free groups in our investigation of strongly semisimple groups. It is possible to generalize Theorem 3.2 for rank one groups to strongly indecomposable groups of finite rank. To do this, we rely heavily on results in [1] and [2].

If $H$ is a subgroup of the torsion free group $G$ such that $G / H$ is a torsion group, then $H$ is a full subgroup of $G$. A subring $S$ of a torsion free ring $R$ is a full subring of $R$ if $S^{+}$is a full subgroup of $R^{+}$. We recall that each torsion free ring $R$ is naturally embedded as a full subring of the rational algebra $Q \otimes_{z} R$ [2].

In the following lemmas, $G$ is a strongly indecomposable torsion free group of finite rank $n$.

LEMmA 5.1. If $R$ is any ring on $G$, then either $\mathscr{J}(R)=\{0\}$ or $R / \mathscr{J}(R)$ is finite.

Proof. It follows frow Theorem 1.4, Corollary 3.6, and Theorem 1.13 in [2], that if $R$ is any ring on $G$, then the rational algebra $Q \otimes_{z} R$ is either nilpotent or is an algebraic number field of dimension $n$ over $Q$. In the first case, $R$ is a nilpotent ring, so that $\mathscr{J}(R)=$ $R$. In the second case, if $I$ is a nonzero ideal in $R$, then $R / I$ is finite [1, p. 206]. Thus, if $Q \otimes_{z} R$ is an algebraic number field, then either $\mathscr{J}(R)=\{0\}$ or $R / \mathscr{J}(R)$ is finite.

A torsion free group $G$ is quotient divisible (or a q.d. group) if $G$ contains a full, free subgroup $F$ such that $G / F$ is divisible. Each torsion free group $G$ of rank $n$ is embedded in a rational vector space $V$ of dimension $n$. Let $\mathscr{L}(V)$ be the ring of all linear transformations of $V$. Then

$$
\mathscr{E}(G)=\{\phi \in \mathscr{L}(V) \mid n \phi(G) \subseteq G \text { for some } n \neq 0 \text { in } Z\}
$$

is a subring of $\mathscr{L}(V)$ called the ring of quasi-endomorphisms of $G$. 
Lemma 5.2. If $G$ is semisimple, then $G$ is a q.d. group, $\mathscr{E}(G)$ is an algebraic number field $K$ such that $[K: Q]=n$, and there is a ring $R$ on $G$ such that $Q \bigotimes_{z} R \cong K$.

Proof. Let $S$ be a (Jacobson) semisimple ring on $G$. If $N$ is the radical of $Q \otimes_{Z} S$, then $N \cap S$ is the maximum nilpotent ideal in $S$, and the rank of $N \cap S$ is equal to the dimension of $N$ [2, p. 71]. Since $N \cap S \leqq \mathscr{J}(S)=\{0\}$, it follows that $N=\{0\}$. Thus, $Q \otimes_{z} S$ is a semisimple algebra. By [2, Corollary 4.9] $G$ is a q.d. group. By [1, Corollary 4.6], $\mathscr{E}(G)$ is an algebraic number field $K$ such that [K: $Q]=n$. Finally, by [1, Theorem 4.1], there is a $\operatorname{ring} R$ on $G$ such that $Q \otimes_{z} R \cong K$.

Lemma 5.3. If $G$ is semisimple, then every ring $R$ on $G$ is isomorphic to a full subring of a single algebraic number field $K$ such that $[K: Q]=n$.

Proof. As in the proof of Lemma 5.1, if $R$ is any ring on $G$, then $Q \otimes_{Z} R$ is either nilpotent or is an algebraic number field $K$ such that $[K: Q]=n$. Wickless [6, Theorem 2.3] shows that it is impossible for both alternatives to hold. By Lemma 5.2, there is a ring $R$ on $G$ such that $Q \bigotimes_{z} R \cong K=\mathscr{E}(G)$. Therefore, by Wickless' result, if $S$ is any ring on $G$, then $Q \otimes_{z} S$ is an algebraic number field $L$ such that $[L, Q]=n$. But by $[1$, Theorem 4.1] $L=\mathscr{E}(G)=K$. That is, every $\operatorname{ring} R$ on $G$ is isomorphic to a full subring of $\mathscr{E}(G)$.

Two subrings $R$ and $S$ of an algebraic number field $K$ are quasiequal $(R \doteq S)$ if there is a positive integer $n$ such that $n R \subseteq S$ and $n S \subseteq R$.

LeMma 5.4. Let $R$ and $S$ be subrings of an algebraic number field $K$ such that $R \doteq S$. Suppose further that $R^{+}$is strongly indecomposable. If $R$ is semisimple, then so is $S$.

Proof. Assume that $S$ is not semisimple. Note that since $R \doteq S$, then $R^{+}$and $S^{+}$are quasi-isomorphic, so that $S^{+}$is strongly indecomposable. Since $\mathscr{J}(S) \neq\{0\}$, it follows from Lemma 5.1, that there is a positive integer $n$ such that $n S \subseteq \mathscr{J}(S)$. Since $R \doteq S$, there is a positive integer $m$ such that $m R \subseteq S$ and $m S \subset R$. Hence $n m R \subseteq$ $n S \subseteq \mathscr{J}(S)$. Since $m S \subseteq R, n m^{2} R \subseteq n m S \subseteq m \mathscr{J}(S)$. Each element of $n m^{2} R$ has a quasi-inverse in the quasi-regular ideal $m \mathscr{J}(S)$ of $S$. Moreover, $m \mathcal{J}(S) \leqq m S \subseteq R$. Thus, each element of $n m^{2} R$ has a quasi-inverse in $R$. But $n m^{2} R$ is an ideal in $R$, hence a quasi-regular ideal. Therefore, $\mathscr{f}(R) \supseteqq n m^{2} R \neq\{0\}$, contradicting the hypothesis 
that $R$ is semisimple.

Let $J$ be the ring of integers in an algebraic number field $K$. In [1] it is shown that the quasi-equality classes of full subrings of $K$ are in one-to-one correspondence with the sets of prime ideals in $J$. If $P$ is any prime ideal in $J$, let $J_{P}=\{x / y \mid x, y \in J, y \notin P\}$. Also, if $\pi$ is any set of prime ideals in $J$, define $J_{\pi}=\bigcap_{P \in \pi} J_{P}$. Then every quasi-equality class of full subrings of $K$ contains one of rings $J_{\pi}, J_{\pi}$ is integrally closed and is the integral closure of every ring in its class. It should be noted that the prime ideals of $J_{\pi}$ are precisely the ideals $P J_{\pi}$ and that nonzero prime ideals in $J_{\pi}$ are maximal. It follows that $\mathscr{J}\left(J_{\pi}\right)=\bigcap_{P \in \pi} P J_{\pi}$.

THEOREM 5.5. Let $G$ be a strongly indecomposable torsion free group of finite rank $n$. Then the following statements are equivalent.

(a) $G$ is semisimple.

(b) $G$ is strongly semisimple.

(c) $G \cong R^{+}$, where $R$ is a full subring of an algebraic number field $K$ such that $[K, Q]=n$, and $R \doteq J_{\pi}$, where $\pi$ is either empty or infinite.

(d) $G$ is nonnil and antiradical.

Proof. By definition, (b) implies (a) and (b) implies (d). We show that (d) implies (b), (a) implies (c), and (c) implies (b).

(d) implies (b). Assume that $G$ is not strongly semisimple. Then $G$ is either a nil group or there is a nonzero ring $R$ on $G$ such that $\mathscr{J}(R) \neq 0$. In the latter case, it follows from Lemma 5.1 that there is a positive integer $m$ such that $m R \subseteq \mathscr{J}(R)$. Since $m R$ is an ideal in $R$, we have $\mathscr{J}(m R)=\mathscr{J}(R) \cap m R=m R$. Hence $m R$ is a nonzero radical ring, so that $m G$ is a radical group. Since $G \cong m G$, it follows that $G$ is a radical group. Thus, if $G$ is not strongly semisimple, then $G$ is either nil or radical.

(a) implies (c). By Lemma 5.3, every ring $R$ for which $G \cong R^{+}$ is a full subring of an algebraic number field $K$ such that $[K, Q]=$ $n$. By the remarks preceding the theorem $R \doteq J_{\pi}$ for some set $\pi$ of prime ideals in $J$. Suppose that $\pi$ is nonempty and finite, and let $\pi=\left\{P_{1}, P_{2}, \cdots, P_{k}\right\}$. Then $\mathscr{J}\left(J_{\pi}\right)=\bigcap_{P \in \pi} P J_{\pi} \supseteqq P_{1} P_{2} \cdots P_{k} J_{\pi} \neq\{0\}$. Hence $J_{\pi}$ is not semisimple. By Lemma 5.4, $R$ is not semisimple. But at least one ring $R$ such that $G \cong R^{+}$is semisimple. For that ring $R, R \doteq J_{\pi}$, where $\pi$ is either empty or infinite.

(c) implies (b). If $\pi$ is the empty set of prime ideals in $J$, then $J_{\pi}=\bigcap_{P \in \pi} J_{P}=K$. Hence $J_{\pi}$ is semisimple. If $\pi$ is an infinite set of 
prime ideals in $J$, then $\mathscr{J}\left(J_{\pi}\right)=\bigcap_{P \in \pi} P J_{\pi}=\{0\}$ since $J_{\pi}$ is a Dedekind ring. Again $J_{\pi}$ is semisimple. Thus, if (c) is satisfied, it follows from Lemma 5.4, that there is a semisimple ring $R$ on $G$. That is, $G$ is semisimple. By Lemma 5.3, every $\operatorname{ring} R$ on $G$ is isomorphic to a full subring of $K$, and hence is quasi-equal to a ring $J_{\pi}$ for some set of prime ideals $\pi$ in $J$. Suppose $R_{1}$ and $R_{2}$ are rings on $G, R_{1} \doteq J_{\pi_{1}}$, $R_{2} \doteq J_{\bar{r}_{1}}, R_{2} \doteq J_{\pi_{2}}$. Then

$$
J_{\pi_{1}}^{+} \doteq R_{1}^{+} \cong R_{2}^{+} \doteq J_{\pi_{2}}^{+},
$$

so that $J_{\pi_{1}}^{+}$is quasi-isomorphic to $J_{\pi_{2}}^{+}$.

Let $\pi^{\prime}$ be the set of rational primes $p$ such that $p \notin P$ for all $P \in \pi$. Then $J_{\pi}^{+}$is $p$-divisible if and only if $p \in \pi^{\prime}$. If $p \in \pi^{\prime}$, then $1 / p \in J_{\pi}$. Hence, if $x / y \in J_{\pi}, x / y=(p x / y)(1 / p)=p(x / y p)$. Therefore, $J_{\pi}^{+}$ is $p$-divisible. On the other hand, if $p \notin \pi^{\prime}, p \in P$ for some $P \in \pi$. If $J_{\pi}$ were $p$-divisible, $1=p(x / y)$ for some $x / y \in J$. But then $y=p x \in$ $P$. This is a contradiction, since if $x / y \in J_{\pi}, y \notin P \in \pi$.

If $G$ and $H$ are quasi-isomorphic torsion free groups, $G$ is $p$ divisible if and only if $H$ is $p$-divisible. Thus, it follows from the result of the preceding paragraph that if $J_{\pi_{1}}^{+}$and $J_{\pi_{2}}^{+}$are quasi-isomorphic, $\pi_{1}^{\prime}=\pi_{2}^{\prime}$. If $\pi_{1}$ is empty, then $\pi_{1}^{\prime}$, and consequently $\pi_{2}^{\prime}$, is the set of all primes. Hence $\pi_{2}$ is empty. If $\pi_{1}$ is infinite, then since each rational prime has only a finite number of prime ideal divisors in $J$, it follows that the complement of $\pi_{1}^{\prime}$ in the set of all primes is infinite. Since $\pi_{1}^{\prime}=\pi_{2}^{\prime}$, the complement of $\pi_{2}^{\prime}$ is infinite. If $\pi_{2}$ were finite, then since each prime ideal $P$ in $J$ contains exactly one rational prime, it follows that $\pi_{2}^{\prime}$ contains almost all primes. But then the complement of $\pi_{2}^{\prime}$ would be finite, a contradiction.

We have shown that if $R$ is any nonzero ring on $G$, then $R \doteq J_{\pi}$, where $\pi$ is either empty or infinite. We have seen that every such $J_{\pi}$ is semisimple. By Lemma 5.4, every nonzero $\operatorname{ring} R$ on $G$ is semisimple. Hence $G$ is strongly semisimple.

\section{REFERENCES}

1. R. A. Beaumont and R. S. Pierce, Subrings of algebraic number fields, Acta Sci. Math. Szeged, 22 (1961), 202-216.

2. - Torsion free rings, Illinois J. Math., 5 (1961), 61-98.

3. L. Fuchs, Abelian Groups, Pergamon Press, New York, 1960.

4. F. Haimo, Radical and antiradical groups, Rocky Mountain J. Math., 3 (1973), 91-106.

5. N. Jacobson, Structure of Rings, Amer. Math. Soc. Colloq. Publications XXXVI.

6. W. J. Wickless, Abelian groups which admit only nilpotent multiplications, Pacific J. Math., 40 (1972), 251-259.

Received May 15, 1973.

UNIVERSITY OF WASHINGTON

UNIVERSITY OF ARIZONA 


\section{PACIFIC JOURNAL OF MATHEMATICS}

\section{EDITORS}

RICHARD ARENS (Managing Editor)

University of California

Los Angeles, California 90024

\section{J. DUGUNDJI}

Department of Mathematics University of Southern California Los Angeles, California 90007

D. Gilbarg and J. Milgram

Stanford University

Stanford, California 94305

University of Washington
Seattle, Washington 98105

ASSOCIATE EDITORS
E. F, BECKENBACH
B. H. NEUMANN
F. WOLF
K. Yoshida

\section{SUPPORTING INSTITUTIONS}

\author{
UNIVERSITY OF BRITISH COLUMBIA \\ CALIFORNIA INSTITUTE OF TECHNOLOGY \\ UNIVERSITY OF CALIFORNIA \\ MONTANA STATE UNIVERSITY \\ UNIVERSITY OF NEVADA \\ NEW MEXICO STATE UNIVERSITY \\ OREGON STATE UNIVERSITY \\ UNIVERSITY OF OREGON \\ OSAKA UNIVERSITY
}

\author{
UNIVERSITY OF SOUTHERN CALIFORNIA \\ STANFORD UNIVERSITY \\ UNIVERSITY OF TOKYO \\ UNIVERSITY OF UTAH \\ WASHINGTON STATE UNIVERSITY \\ UNIVERSITY OF WASHINGTON \\ * * * * \\ AMERICAN MATHEMATICAL SOCIETY \\ NAVAL WEAPONS CENTER
}

The Supporting Institutions listed above contribute to the cost of publication of this Journal, but they are not owners or publishers and have no responsibility for its content or policies.

Mathematical papers intended for publication in the Pacific Journal of Mathematics should be in typed form or offset-reproduced, (not dittoed), double spaced with large margins. Underline Greek letters in red, German in green, and script in blue. The first paragraph or two must be capable of being used separately as a synopsis of the entire paper. Items of the bibliography should not be cited there unless absolutely necessary, in which case they must be identified by author and Journal, rather than by item number. Manuscripts, in duplicate if possible, may be sent to any one of the four editors. Please classify according to the scheme of Math. Rev. Index to Vol. 39. All other communications to the editors should be addressed to the managing editor, or Elaine Barth, University of California, Los Angeles, California, 90024.

100 reprints are provided free for each article, only if page charges have been substantially paid. Additional copies may be obtained at cost in multiples of 50 .

The Pacific of Journal Mathematics is issued monthly as of January 1966. Regular subscription rate: $\$ 72.00$ a year (6 Vols., 12 issues). Special rate: $\$ 36.00$ a year to individual members of supporting institutions.

Subscriptions, orders for back numbers, and changes of address should be sent to Pacific Journal of Mathematics, 103 Highland Boulevard, Berkeley, California, 94708.

PUBLISHED BY PACIFIC JOURNAL OF MATHEMATICS, A NON-PROFIT CORPORATION

Printed at Kokusai Bunken Insatsusha (International Academic Printing Co., Ltd.), 270, 3-chome Totsuka-cho, Shinjuku-ku, Tokyo 160, Japan.

Copyright (C) 1973 by Pacific Journal of Mathematics Manufactured and first issued in Japan 


\section{Pacific Journal of Mathematics}

\section{Vol. 53, No. $2 \quad$ April, 1974}

Kenneth Abernethy, On characterizing certain classses of first countable spaces by

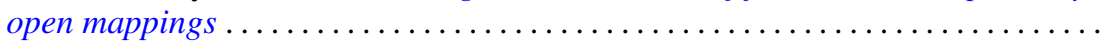

Ross A. Beaumont and Donald Lawver, Strongly semisimple abelian groups .......

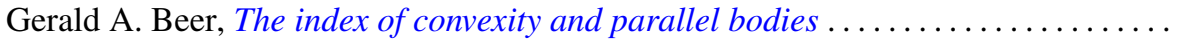

Victor P. Camillo and Kent Ralph Fuller, On Loewy length of rings ..............

Stephen LaVern Campbell, Linear operators for which $T^{*} T$ and $T T^{*}$ commute.

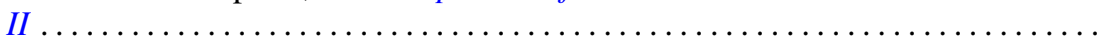

Charles Kam-Tai Chui and Philip Wesley Smith, Characterization of a function by

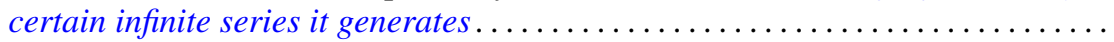

Allan L. Edelson, Conjugations on stably almost complex manifolds . ...........

Patrick John Fleury, Hollow modules and local endomorphism rings . . ..........

Jack Tilden Goodykoontz, Jr., Connectedness im kleinen and local connectedness in

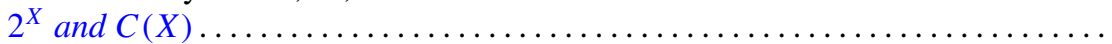

Robert Edward Jamison, II, Functional representation of algebraic intervals .......

Athanassios G. Kartsatos, Nonzero solutions to boundary value problems for

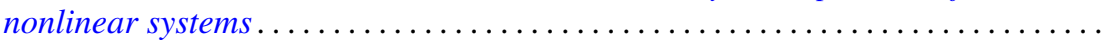

Soon-Kyu Kim, Dennis McGavran and Jingyal Pak, Torus group actions on simply

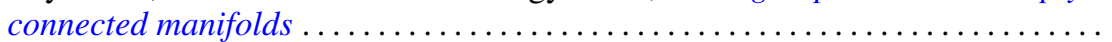

David Anthony Klarner and R. Rado, Arithmetic properties of certain recursively

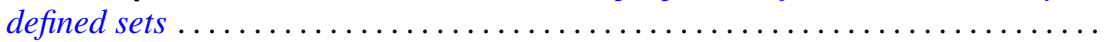

Ray Alden Kunze, On the Frobenius reciprocity theorem for square-integrable

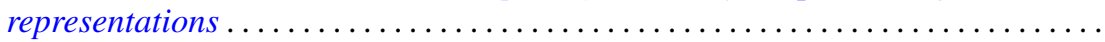

John Lagnese, Existence, uniqueness and limiting behavior of solutions of a class of differential equations in Banach space...

Teck Cheong Lim, A fixed point theorem for families on nonexpansive mappings Lewis Lum, A quasi order characterization of smooth continua

Andy R. Magid, Principal homogeneous spaces and Galois extensions . .

Charles Alan McCarthy, The norm of a certain derivation ..... . .

Louise Elizabeth Moser, On the impossibility of obtaining $S^{2} \times S^{1}$ by elementary surgery along a knot. .

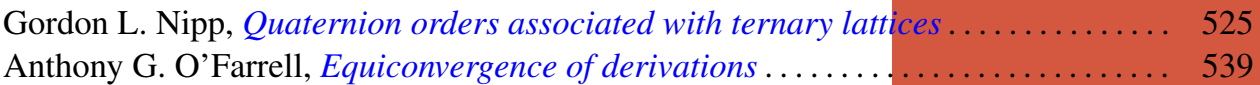

Dorte Olesen, Derivations of $A W^{*}$-algebras are inner . . . . . . . . . . . . . . . 555

Dorte Olesen and Gert Kjærgaard Pedersen, Derivations of $C^{*}$-algebras have

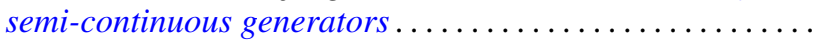

Duane O’Neill, On conjugation cobordism.

Chull Park and S. R. Paranjape, Probabilities of Wiener paths crossing differentiable

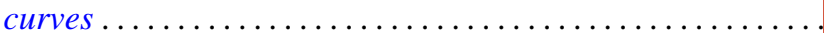

Edward Ralph Rozema, Almost Chebyshev subspaces of $L^{1}(\mu$;

Lesley Millman Sibner and Robert Jules Sibner, A note on the Atiyah-Bott fixed

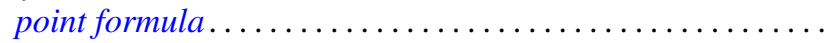

Betty Salzberg Stark, Irreducible subgroups of orthogonal groups generated by

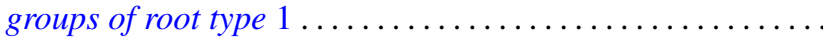

N. Stavrakas, A note on starshaped sets, $(k)$-extreme points and the half ray

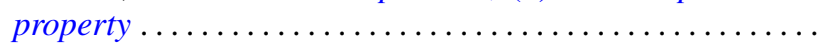

Carl E. Swenson, Direct sum subset decompositions of $Z \ldots \ldots$ 